\title{
Feature Extraction of Bearing Status Based on Multi-Scale Bistable Stochastic Resonance Array
}

\author{
Xiaofei Zhang, Jingwei Gao, Lijun Song, Shuming Yang \\ College of Basic Education, National University of Defense Technology, Changsha, 410073, China \\ zhangxiaofei@nudt.edu.cn
}

Keywords: Multi-Scale Bistable Array; Stochastic Resonance; Bearing; Feature Extraction

\begin{abstract}
Multi-scale bistable array (MSBA), which combines normalized scale transform, stochastic resonance effect driven by colored noise and parallel array, can be applied to weak signal detection under heavy noise. The experimental application in incipient fault feature detection of rolling element bearing has verified the effectiveness of MSBA model. This paper studies feature extraction method of rolling element bearing status degradation based on enhanced detection effect of MSBA model. Integrated features are proposed using local spectrum kurtosis and local signal-to-noise ratio of fundamental component of bearing faults. Different sizes of damages are planted on outer race of bearings for experimental validation.
\end{abstract}

\section{Introduction}

An effective prognostics and health management (PHM) system is expected to provide not only early detection of incipient fault of important components but also the means to monitor and predict the progression of the fault[1]. Rolling element bearing, as a critical component of transmission, is widely used in mechanical equipment. Features which indicate bearing status degradation progress can be used in forecasting, or remaining useful life prediction. However, noise has often stunted the feature extracting procedures.

Since stochastic resonance (SR) was proposed, it has been developing rapidly in incipient fault detection [2-9], especially in low signal-to-noise ratio (SNR) cases. It is essentially a statistical phenomenon resulting from an effect of noise on information transfer and signal processing which is observed in both man-made and naturally existing nonlinear systems. Compared with conventional linear methods, the methods based on SR are promising in the conditions of short data records and heavy noise.

This paper focuses on feature extracting method of bearing status degradation based on multi-scale bistable array (MSBA), which is a stochastic resonance model proposed for weak signal detection used in incipient fault diagnosis [9]. The bearing vibration signal is processed by signal processing method based on MSBA. Local spectral kurtosis (LSK) and local signal-to-noise ratio (LSNR) at bearing fault frequencies are proposed as indicators of bearing status. Combined with RMS and time domain statistical characteristics, LSK and LSNR are treated as the subset of bearing status feature vector. Finally, new integrated features are constructed for incipient fault detection and status degradation monitoring purpose.

\section{Basic Theory of Multi-Scale Bistable array}

In the context, stochastic resonance is commonly described as an approach to increase the SNR at the output through the increase of the special noise level at input signal. Considering the motion in a bistable double-well potential of a lightly damped particle subjected to stochastic excitation and a harmonic excitation (i.e., a signal) with low frequency $\omega_{0}$, the signal is assumed to have small enough amplitude that, by itself (i.e., in the absence of the stochastic excitation), is unable to move the particle from one well to another. We denote the characteristic rate, that is, the escape rate from a well under the combined effects of the periodic excitation and the noise, by $\alpha=2 \pi n_{\text {tot }} / T_{\text {tot }}$, where $n_{\text {tot }}$ is the total number of exits from one well during time $T_{\text {tot }}$. We consider the behavior of the 
system as we increase the noise while the signal amplitude and frequency are unchanged. For zero noise, $\alpha=0$, as noted earlier. For very small noise, $\alpha<\omega_{0}$. As the noise increases, the ordinate of the spectral density of the output noise at the frequency $\omega_{0}$, denoted by $\Phi_{n}\left(\omega_{0}\right)$, and the characteristic rate $\alpha$ increases. Experimental and analytical studies show that, until $\alpha \approx \omega_{0}$, a cooperative effect (i.e., a synchronization-like phenomenon) occurs wherein the signal output power $\Phi_{s}\left(\omega_{0}\right)$ increases as the noise intensity increases. Remarkably, the increase of $\Phi_{s}\left(\omega_{0}\right)$ with noise is faster than that of $\Phi_{n}\left(\omega_{0}\right)$. This results in an enhancement of the SNR.

At present, the most common studied SR system is bistable system, which can be described by the following Langevin equation

$$
\dot{x}=a x-b x^{3}+A \sin \left(\omega_{0} t+\varphi_{0}\right)+\Gamma(t)
$$

$\Gamma(t)$ is noise term and $<\Gamma(t), \Gamma(0)>=2 D \delta(t)$, Asin $\left(\omega_{0} t+\varphi_{0}\right)$ is a periodic driving signal. Generally, it is also written as the form of Duffing equation

$$
\ddot{x}=-\beta \dot{x}+a x-b x^{3}+A \sin \left(\omega_{0} t+\phi_{0}\right)+\Gamma(t)
$$

where $\beta$ is the damping coefficient.

The SR effect is still shown in a nonlinear bistable system when the white noise is changed to band-limited noise, which indicates that it is possible to realize the SR by tuning the band-limited noise [9, 10]. To improve the signal processing based on SR when the original noise intensity is beyond the optimal level, the input signal is decomposed into multi-scale signals by orthogonal wavelet transform. Stationary white noise with zero mean can be decomposed into independent band-limited noises by orthogonal wavelet transform. The reconstructed detail at each scale and the approximation signal at the last scale are independent of each other owing to the orthogonality of wavelet base.

By combining uncoupled parallel array of dynamical subsystems with colored noise SR effect, an MSBA consisting of bistable units formulated as (1) is constructed. Figure 1 illustrates the configuration of the MSBA. The input signal is decomposed into different scale signals by wavelet transform. In the context, $a_{j}$ and $d_{j}$ denote reconstructed approximation signals and detail signals, respectively for convenience. The driving signal of the MSBA, which is in the low frequency region, is supposed to be contained in the approximate signal $a_{J}$. The approximate signal $a_{J}$ and each scale noise $d_{j}$ reconstruct the new input signal of each bistable element. Then, the number of array elements is equal to the scale number $J$. Being uncoupled between any two elements, the outputs of all units are averaged together to produce the entire array output $y(t)$. Similar to uncoupled parallel SR array, each element is subjected to an independent array noise $d_{j}$ and the same noisy input signal $a_{J}$. However, the inner array noise intensity and characteristics of the MSBA are different from those of the uncoupled parallel array. The noise intensity of input signal is reduced after decomposition. The noise at different scale has a different contribution to the SR effect of the single bistable element in the array.

Numerical simulation is performed according to the MSBA model given in Figure 1. Each bistable element in the MSBA is formulated as (1). The SR effect of the MSBA is evaluated with tuning the input noise intensity $D$ and the analyzed scale number $J$. The other system parameters are chosen as $a=b=1, A_{0}=0.3, f_{0}=0.01 \mathrm{~Hz}$ and $\varphi=0$. The sampling frequency is set to be $f_{\mathrm{s}}=500 f_{0}=5 \mathrm{~Hz}$, and the data length of the input signal is set to be 4000 .

Figure 2 shows the SR effect of the MSBA with tuning noise intensity $D$ and the analyzed scale number $J$. The array output SNR curves, from bottom up, correspond to $J=1,2,3,4$, 5, and 6 , respectively. The other parameters are chosen as $a=b=1, A_{0}=0.3, f_{0}=0.01 \mathrm{~Hz}$ and $\varphi=0$. The results indicate that the tuning noise intensity $D$ of the input signal produces an obvious SR effect on the MSBA. When the value of $D$ increases, the array output SNR first increases and then decreases after reaching a maximum. It can be found that the output SNR resonance region is broadened with the increase of the analyzed scale number $J$, and also the maximum point is moved to a larger noise intensity $D$ with almost the same value. When the noise intensity $D$ becomes larger, the SNR curves of $J>1$ will go up compared with the curve of $J=1$. This means that the output signal of the MSBA has been enhanced further than that of the single bistable system at a larger input noise intensity $D$. 
Thus, the model of MSBA has admirable capability in signal processing based on SR under large noise.
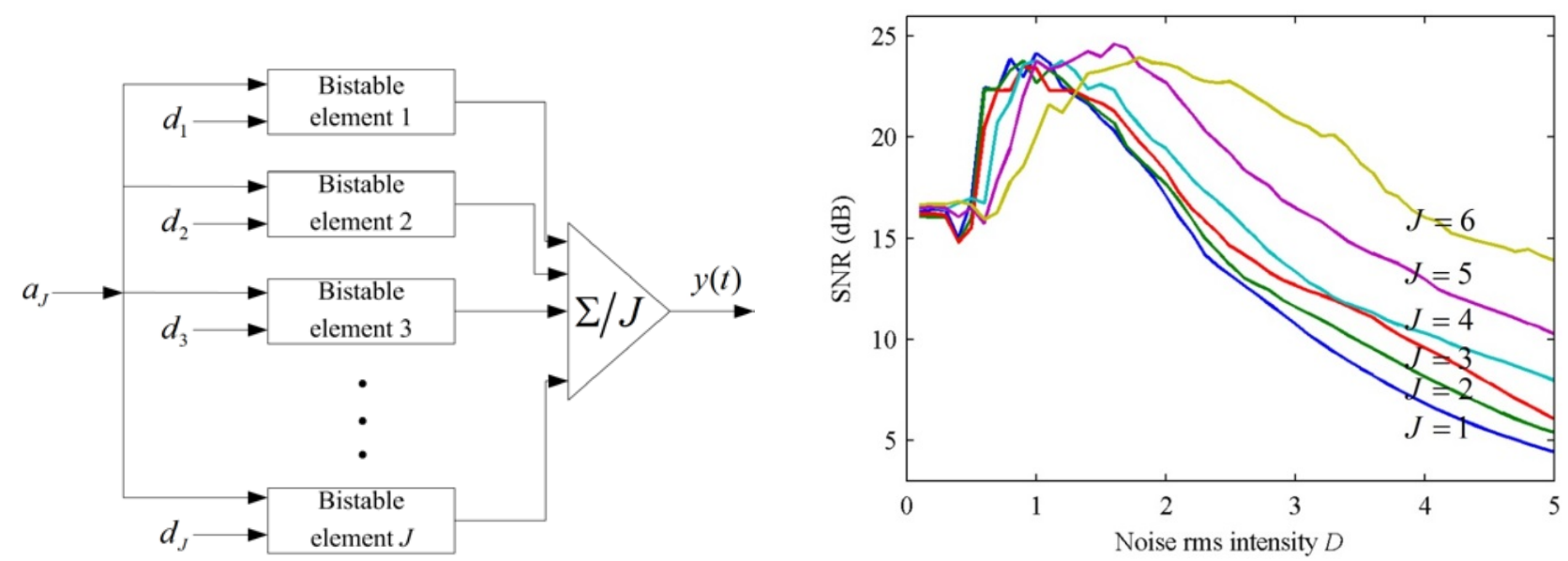

Fig.1. MSBA model of $J$ elements. Fig.2. Output SNR of the MSBA versus tuning noise intensity $D$ and analyzed scale number $J$

\section{Feature extraction method based on MSBA model}

Enhanced detection method based on MSBA has been applied to incipient fault diagnosis [9]. In this paper, vibrational features extracted at bearing fault frequencies are used to indicate bearing status degradation. Local spectral kurtosis and local signal-to-noise ratio around bearing fault frequencies is calculated not only for fault diagnosis but also for status tracking. Integrated features are extracted based on the time domain characteristics and enhanced features, LSK and LSNR exactly. The integrated features can be used to fault diagnosis and progression monitoring.

Assume that the vibration signal is $x(n)$. Then, root mean square of $x(n)$ is

$$
R M S=\sqrt{\frac{1}{N} \sum_{n=1}^{N} x^{2}(n)}
$$

$N$ is the data length of $x(n)$.

Crest factor of $x(n)$ is calculated as

$$
C F=\frac{\hat{x}}{R M S}
$$

$\hat{x}$ is the peak value of $x(n)$.

Kurtosis of $x(n)$ is

$$
K=\frac{\sum_{n=1}^{N}[x(n)-\mu]^{4}}{N\left(\sigma^{2}\right)^{2}}
$$

$\mu$ is the mean value of $x(n), \sigma^{2}$ is the variance of data, $N$ is the data length.

The vibration signal from bearing is processed, during which the component at fault frequencies are enhanced, by weak signal detection method based on the classical SR and MSBA model. The local spectral kurtosis is defined as kurtosis of spectral data around bearing fault frequencies. For given length spectral data $X(f)$, which cover bearing fault frequency component, the LSK is calculated as

$$
\operatorname{LSK}(f)=\frac{\left\langle|X(f)|^{4}\right\rangle}{\left\langle|X(f)|^{2}\right\rangle^{2}}
$$

$X(f)$ is a section of bearing envelope spectrum enhanced by SR or MSBA model.

Similarly, the LSNR is calculated as

$$
L S N R=\lim _{\Delta f \rightarrow 0}\left(\int_{f-\Delta f}^{f+\Delta f}\left[S(f) / S_{N}(f)\right] \mathrm{d} f\right)
$$

$S(f)$ is the value of spectral component at bearing fault frequency, $S_{N}(f)$ is the mean value of the 
spectrum around bearing fault frequency.

Due to noise from measurement and other dynamical components, it is difficult to indicate bearing status using only one of the features mentioned above. Additionally, damage position cannot be figured out based on the time domain characteristics. The enhanced frequency domain features is suitable for incipient fault diagnosis but inappropriate to bearing status tracking. So, traditional features are combined with frequency domain features enhanced by MSBA, as depicted in Figure 3.

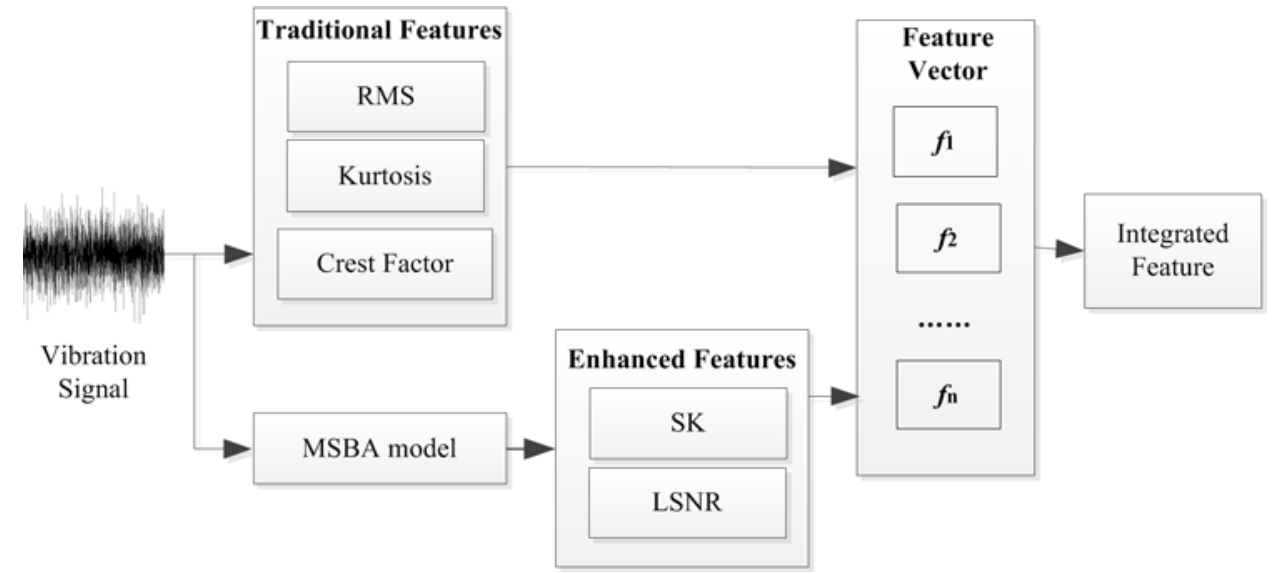

Fig.3. Diagram of integrated feature extraction method

All the features extracted are standardized to form a feature vector

$$
\boldsymbol{F}=\left[A_{1} \frac{R M S}{R M S_{\max }}, A_{2} \frac{C F}{C F_{\max }}, A_{3} \frac{K}{K_{\max }}, A_{4} \frac{S K}{S K_{\max }}, A_{5} \frac{L S N R}{L S N R_{\max }}\right]
$$

$A_{i}$ is weighted coefficients, $i=1,2,3,4,5$. The integrated feature of the vector is calculated as

$$
I=\|F\|=\left(\sum_{i=1}^{n}\left|f_{i}\right|^{2}\right)^{\frac{1}{2}}
$$

\section{Test results}

The proposed extraction method is applied to signal from machinery fault simulation test rig shown in Figure 4. Tests were carried out on the test rig with good and planted-in outer race fault bearings. The test rig is driven by a variable-speed electric motor. For these tests, the motor was running at 660 RPM, with two rotor disks on the shaft. The Bearing1 in Figure 4 is alternated with normal bearing, bearing with 0.2-3.2 mm different size outer race faults, which are shown in Figure 5 . The raw vibration data were collected with the sampling rate $50 \mathrm{kHz}$.

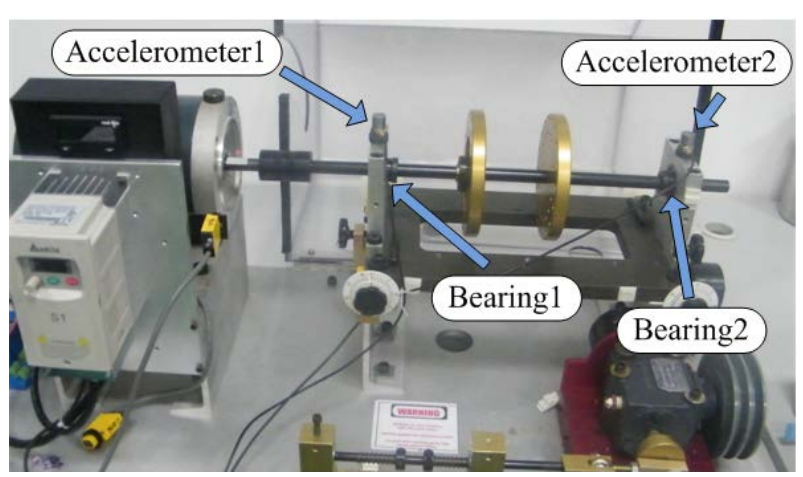

Fig.4. Machinery fault simulation test rig
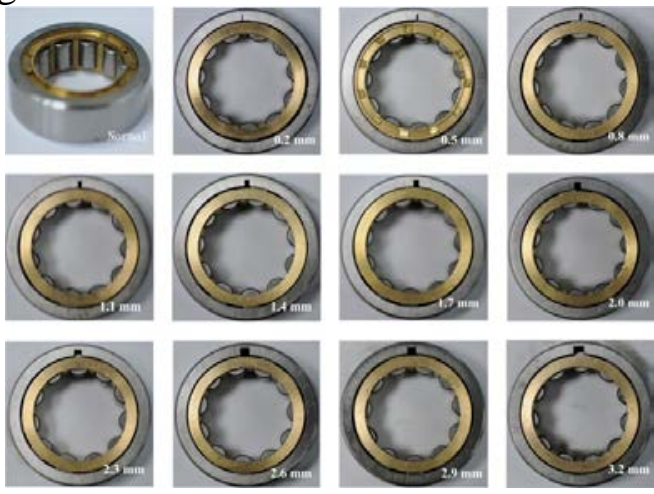

Fig.5. The outer races used in the experiments

The time domain features are calculated using the vibration data collected on working bearings with different sizes of outer race damages. Figure 6 displays the RMS feature curve of the bearings tested. The curve shows that the RMS feature has strong rising trend with increasing of outer race fault size, though there are still some fluctuations. But we cannot distinguish fault bearing from the normal one apparently when the fault size is small. 
The kurtosis feature curve is depicted in Figure 7. The curve also has a rising trend with increasing of outer race fault size and also some fluctuations, especially when the fault size is greater than $1.1 \mathrm{~mm}$. We still cannot identify fault bearing easily at the beginning of the curve.

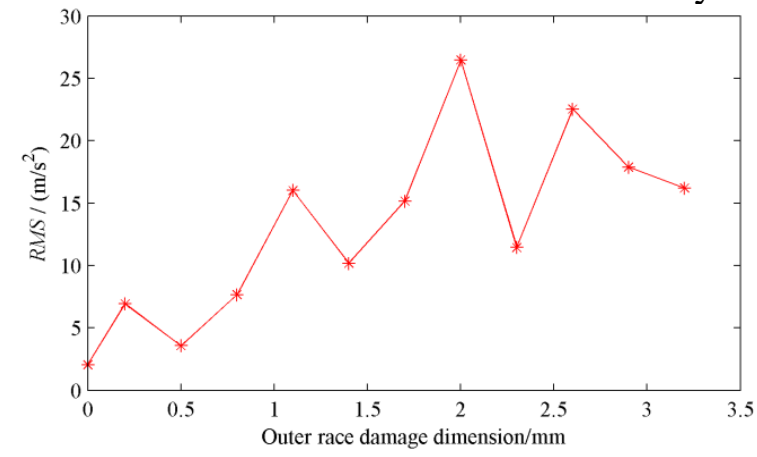

Fig.6. The curve of RMS feature

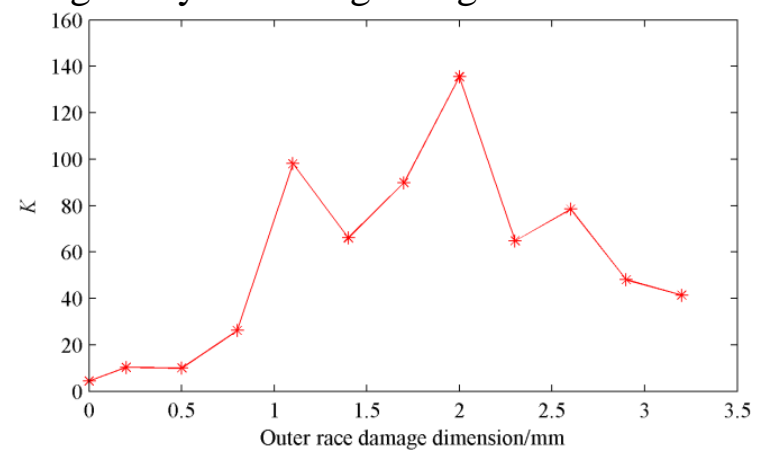

Fig.7. The curve of kurtosis feature

The crest factor feature curve is depicted in Figure 8. The curve has a smooth rising trend with increasing of fault size and some fluctuations, especially when the fault size is greater than $1.4 \mathrm{~mm}$. It will be misleading if the crest factor is used to diagnose $0.2 \mathrm{~mm}$ outer race bearing fault.

The vibration signal was processed by signal enhanced method based on traditional SR and MSBA model. Then, the LSK and LSNR features are calculated according to (6) and (7) around outer race fault frequency in frequency domain. The curves are shown in Figure 9 and Figure 10. There are similar uptrends in the two feature curves. The feature curves processed by MSBA model are more sensitive to incipient bearing fault than that processed by traditional SR model.

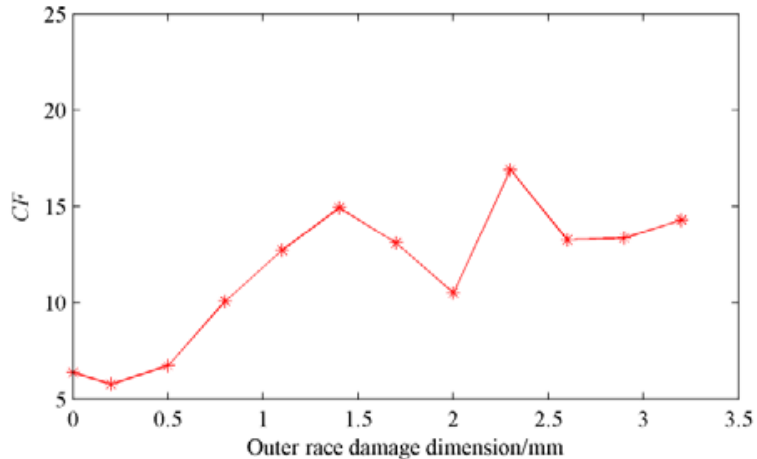

Fig.8. The curve of crest factor

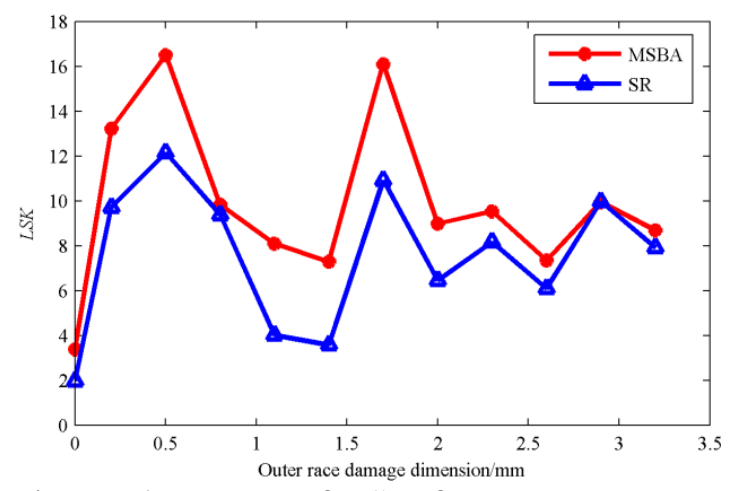

Fig.9. The curve of LSK features

Any one of the above features cannot be used in bearing status tracking for the existence of intensive fluctuations of the curves. Meanwhile, time domain features cannot locate the bearing fault position. Feature vector is calculated according to (8). And the two similar integrated features using LSK and LSNR based on MSBA model are shown in Figure 11. Despite some light fluctuations, there is a steep uptrend and an obvious distinction between normal and fault bearings. The integrated features have potential in the application of both fault diagnosis and status tracking when the bearing fault size is smaller than $2 \mathrm{~mm}$. Once the damage size is larger than $2 \mathrm{~mm}$, the integrated features have a down trend and the bearing may cause downtime in a short time.

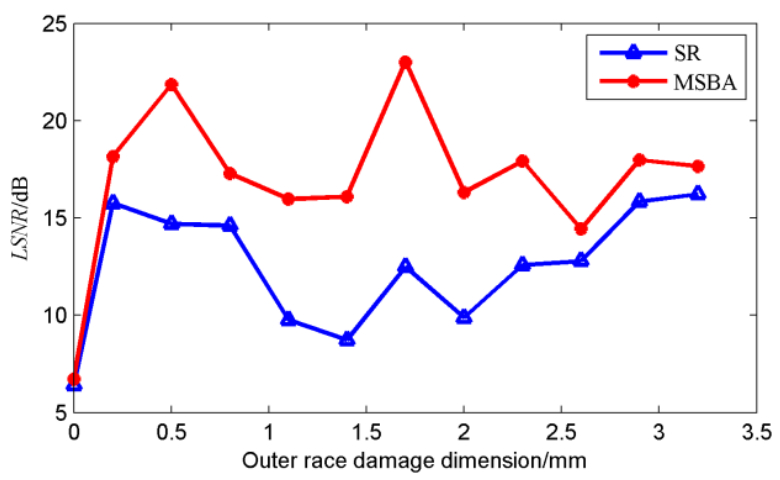

Fig.10. The curve of LSNR features

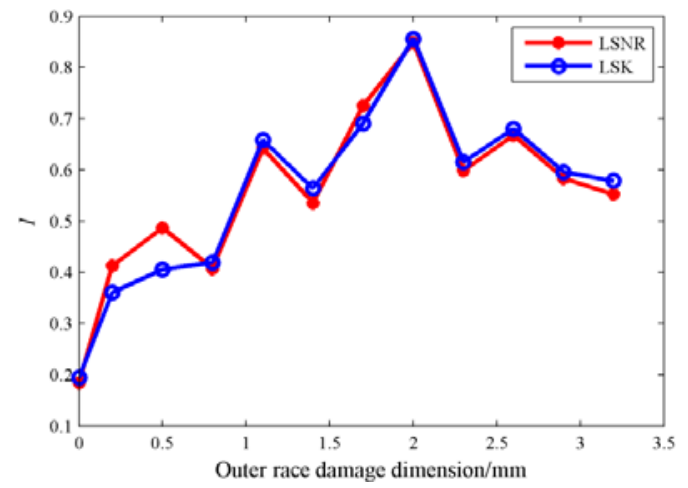

Fig.11. The curve of integrated features 


\section{Conclusion}

For vibration signal of bearing, the time domain feature curves have uptrends with fluctuations when bearing damage size is growing up. The LSK and LSNR features enhanced by MSBA model have advantages in incipient bearing fault diagnosis. The integrated features proposed in this paper can be used in both fault diagnosis and status tracking. The conclusions above are validated with outer race fault experiment.

\section{Acknowledgement}

In this paper, the research was sponsored by the National Science Foundation of China (51405500), Pre-research Foundation (9140A17040114KG01242) and Science Foundation of National University of Defense Technology (ZK16-03-14 and JC14-09-01).

\section{References}

[1] Jay Lee, Fangji Wu, Wenyu Zhao, Masoud Ghaffari, Linxia Liao and David Siegel. Prognostics and health management design for rotary machinery systems-Reviews, methodology and applications[J], Mechanical Systems and Signal Processing, 2014 (44) 314-334.

[2] ZHANG Xiaofei, HU Niaoqing, CHENG Zhe, HU Lei. Enhanced Detection of Rolling Element Bearing Fault Based on Stochastic Resonance[J], Chinese Journal of Mechanical Engineering, 2012 25(6) 1287-1297.

[3] LAI Xin-huan, ZHAO Jun, KONG Ming, et al. Weak feature extraction of gear fault based on Stochastic Resonance denoising [C], Eighth International Symposium on Precision Engineering Measurement and Instrumentation, 2013.

[4] Bing Li, Jimeng Li, Jiyong Tan, et al. AdSR Based Fault Diagnosis for Three-Axis Boring and Milling Machine[J], Journal of Mechanical Engineering, 2012 58(9) 527-533.

[5] Jimeng Li, Xuefeng Chen, Zhengjia He. Adaptive stochastic resonance method for impact signal detection based on sliding window[J], Mechanical Systems and Signal Processing, 2013 (36) 240-255.

[6] Dongying Han, Peili, Shujun An, Peiming Shi. Multi-frequency weak signal detection based on wavelet transform and parameter compensation band-pass multi-stable stochastic resonance[J], Mechanical Systems and Signal Processing, 2016 (70) 995-1010.

[7] Siliang Lu,Qingbo He, Fanrang Kong. Effects of underdamped step-varying second-order stochastic resonance for weak signal detection[J], Digital Signal Processing, 2015 (36) 93-103.

[8] Xi-hui Chen, Gang Cheng, Xian-lei Shan, et al. Research of weak fault feature information extraction of planetary gear based on ensemble empirical mode decomposition and adaptive stochastic resonance[J], Measurement, 2015 (73) 55-67.

[9] ZHANG Xiaofei, HU Niaoqing, HU Lei, CHENG Zhe. Multi-scale bistable stochastic resonance array: A novel weak signal detection method and application in machine fault diagnosis[J], Science China Technological Science, 2013 56(9) 2115-2123.

[10] Gammaitoni, L., Hanggi, P., Jung, P., et al. Stochastic resonance[J], Reviews of Modern Physics, 1998 70(1) 223-287. 